\section{X-Ray Beam Segment Size and Entrance Location Effects on the Integral Quality Monitor (IQM®) Signal and Usefulness in Predicting Complex Segment Output Signals}

\author{
Mahuvava C. ${ }^{1 \oplus}$, Du Plessis F. C. P. ${ }^{1 *(0)}$
}

\begin{abstract}
Background: The Integral Quality Monitor (IQM $\left.{ }^{\circledR}\right)$ is an independent online dosimetry device attached to the treatment machine to monitor the accuracy of radiation delivery.

Objective: This study investigates the influence of beam segment size and displacement as projected onto the IQM chamber on the signals and determine how individual signals can be added to get a combined segment signal made up of smaller segments.
\end{abstract}

Material and Methods: This is an experimental original research type of study. IQM response maps were generated by irradiating the IQM sensitive area with small elementary segments and measuring their corresponding signals per monitor unit (MU). The output signal/MU was measured for regular and irregular fields and compared with the predicted signal/MU obtained from decomposing the open segment into a set of smaller regular segments and summing their signals from their respective response maps. The dependence of signals on segment size, shape, location and combination was investigated.

Results: Predicted signals were calculated within 95-98\% accuracy for regular fields and $90-98 \%$ for irregular fields. More uniform fluence contain distribution for larger segments was observed. Response maps were consistent with the geometrical symmetry in the chamber's wedge shape and the symmetry in the linac fluence.

Conclusion: The field decomposition method allows the pre-calculation of known segment output signals per MU within 2\% error, although the accuracy drops significantly for smaller, irregular fields. A method of correcting predicted signals in smaller segments needs to be laid down to get a better match with measured signals.

Citation: Mahuvava C, Du Plessis FCP. X-Ray Beam Segment Size and Entrance Location Effects on the Integral Quality Monitor (IQM $\mathbb{B})$ Signal and Usefulness in Predicting Complex Segment Output Signals. J Biomed Phys Eng. 2020;10(4):395-410. doi: 10.31661/jbpe.v0i0.1162

\section{Keywords}

Integral Quality Monitor; Output Signal; Response Map; Fluence

\section{Introduction}

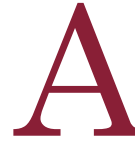
$\mathrm{s}$ radiotherapy systems and delivery techniques have been becoming more intricate, the human factor and its contribution to the detection and prevention of errors is becoming less effective during radiation treatment [1]. This complexity can be broken by
${ }^{1} \mathrm{PhD}$, Department of Medical Physics, Faculty of Health Sciences, University of the Free State, P.O. Box 339, Bloemfontein, 9300 South Africa
*Corresponding author:

F. C. P. Du Plessis

Department of Medi-

cal Physics, Faculty of

Health Sciences, Uni-

versity of the Free State,

P.O. Box 339, Bloemfon-

tein, 9300 South Africa

E-mail: DuPlessisFCP@

ufs.ac.za

Received: 15 April 2019 Accepted: 12 June 2019 
placing a transmission detector encompassing the entire radiation field on the linac head and performing dosimetric measurements of the radiation beams as they are delivered to the patient. Online beam monitoring has the potential to detect many probable treatment delivery errors [2].

Several research groups have devised or investigated various real-time beam monitoring systems. These include the DAVID ${ }^{\circledR}$ system $[3,4]$, the COMPASS $®$ system [5-8], the VANILLA system [9], the 'magic plate' [10] as well as the optical fibre based system proposed by Goulet, Gingras and Beaulieu [11]. The IQM system is interested [1, 12], which is an independent online dosimetry system attached to the treatment machine to verify the integrity and accuracy of treatment delivery and capture common error conditions while permitting the unperturbed transmission of the radiation intensity pattern to the patient [13].

The IQM utilizes a novel large area ionization chamber to measure the dose-area-product (DAP) for each beam segment and validate the accuracy of energy fluence in real time. It is capable of picking up errors related to dose, patient details, individual beam size and direction, MLC leaf calibration and positioning, beam segment shape and intensity maps. The prototype beam delivery check system consists of an area integrating fluence monitoring sensor (AIMS) positioned between the MLC and the patient, and an independent software platform configured to calculate the expected signal. The expected signal is calculated by the IQM calculation module based on sector integration of the chamber signals corresponding to the beam energy fluence [14]. During beam delivery, the expected signal is compared to the measured signal and warnings are issued when significant deviance occurs.

The dual wedge-shaped aluminium chamber has a spatial gradient in the electrode plate separation along the direction of the MLC motion and a dosimetry system incorporating a wide dynamic range switching electrometer to capture the energy fluence for each beam segment. The single-channel detector is connected wirelessly to a transceiver and the controlling computer. A gradient in the chamber's active volume thickness creates spatial sensitivity in the gradient direction (i.e., makes the magnitude of the signal dependent on the beam position) $[15,16]$. According to Islam et al. [1], SAIMS (the AIMS signal for a segment) is given by equation 1 :

$\mathrm{S}_{\mathrm{AIMS}}=M U \cdot K \cdot A O F(x, y) \cdot\left[\int_{A 1}^{1} F(x, y) \sigma(x, y) d x d y+\int_{A-A 1}^{1} T_{M L C}(x, y) F(x, y) \sigma(x, y) d x d y+\int_{R-A}^{1} T_{\text {Jaw }}(x, y) T_{M L C}(x, y) F(x, y) \sigma(x, y) d x d y\right](1$

Where MU is the monitor unit, which is set for the segment, $\mathrm{K}$ is the system constant and $A O F(x, y)$ is the output change with field size. $F(x, y)$ represents the fluence distribution and $\sigma(x, y)$ is the chamber spatial response function describing the relative chamber response for an elementary segment at position $(x, y)$. The response function includes the chamber sensitivity and off-axis beam intensity variation. The limits of the integral A1 and A refer to the effective aperture regions defined by the MLC and jaws. The third term in equation 1 accounts for the signal component due to jaw and/or MLC leakage to the chamber with area R. $T_{J a w}$ and $T_{M L C}$ define the corresponding average transmission factors through MLCs and jaws.

The IQM electronic signal is a measure of the linac radiation output transmitted through the chamber [17]. This study aims to quantitatively determine the influence of beam segment size and displacement as projected onto the IQM chamber on the signals and to determine how individual signals can be added to get a combined segment signal made up of smaller segments. However, The IQM cannot predict the dose in a patient, it (in principle) can measure the integral fluence through a segment exiting the linac. This study determines if it is possible to predict the signal/MU for an arbitrary segment if its response is known for a series of smaller regular segments that 
comprise the arbitrary segment. This, in principle, would be allowed for the pre-calculation of known irregular segment output signals per MU, which will be useful for patient based dose calculation for treatment verification during pre-treatment IMRT treatment validation.

\section{Material and Methods}

This is an experimental original research type of study. The research was approved by the Health Sciences Research Ethics Committee; ethics clearance number: UFSHSD2018/0019/2808. The study did not contain any human or animal subjects.

IQM signals are influenced by temperature, pressure, number of MUs, scattering radiation from the linac head structure, beam energy and segment shape. Since the IQM is wedged shaped, the location of the beam portal (segment) as projected onto the chamber will also influence the IQM signal. Similar segments can give different signals due to the chamber's wedge shape and machine output variation across the field. Different segments can also give the same signal. It is thus necessary to know the exact segment shape and location on the chamber plane in order to predict the output signal per MU for further dose calculations.

\section{Determining IQM response maps with elementary (regular) segments}

The first part of the study was to generate several response maps of the IQM chamber for various elementary small regular segment sizes and beam energies. The IQM was attached to the accessory holder of an Elekta Synergy linac equipped with Agility 160-leaf MLCs. The response map of $1 \times 1 \mathrm{~cm}^{2}$ segment for 35 MUs per segment set on the linac was measured. The response maps for $2 \times 2,3 \times 3,5 \times 5$ and $7 \times 7 \mathrm{~cm}^{2}$ segments were also measured, shifting the segment $1 \mathrm{~cm}$ at a time (both in the gradient $(x)$ and non-gradient $(y)$ directions as shown in Figure 1) and measuring the corresponding signal/MU for each regular, shifted segment. This gave 2D spatial response maps over the IQM wedge-shaped chamber sensitive area for these sets of shifted regular segments.

Since the IQM has geometric symmetry along the wedge gradient direction, it is pos-

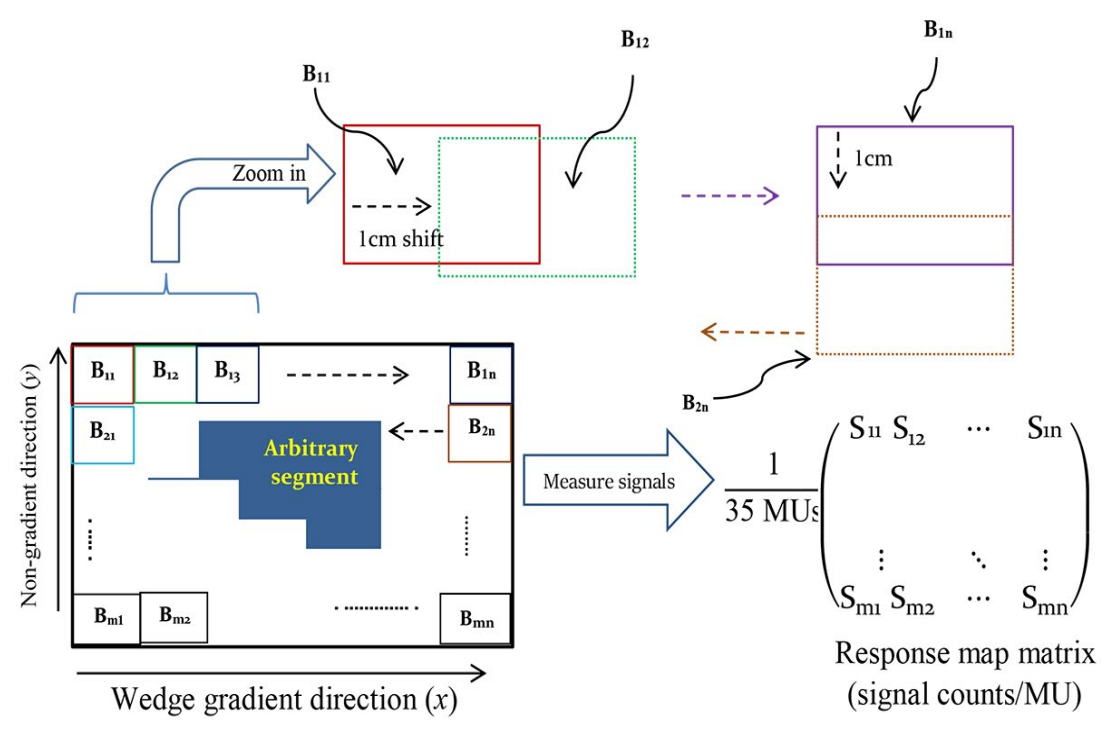

Figure 1: Planar view of the IQM irradiated with small (overlapping) segments denoted by $B_{11^{\prime}}$ $B_{12}, B_{13} \ldots$ Bmn and a corresponding DAP signal $\left(S_{11}, S_{12} \ldots S_{m n}\right)$ per MU measured for each segment to create a signal matrix/response map of the entire IQM sensitive area. The chamber has a sensitive area of $\sim 26 \times 26 \mathrm{~cm}^{2}$. 
sible to measure half of the fluence map and reflect it along the symmetry axis. However, this reflection process may present problems when the field edges of the elementary segments nearest to the IQM gradient symmetry axis are not aligned with the symmetry axis i.e., when they cross or do not reach the symmetry axis. As a result, there could be a gap or an overlap between the original and the reflected response map. For this reason, this technique was not used.

In Figure 1, segment $\mathbf{B}_{11}$, was shifted $1 \mathrm{~cm}$ at a time in the $+x$ direction until reached the far end of the chamber sensitive area at $\mathbf{B}_{1 \mathrm{n}}$ $\mathbf{B}_{1 \mathrm{n}}$ was then shifted $1 \mathrm{~cm}$ in the $-y$ direction (down) to position $\mathbf{B}_{2 \mathrm{n}}$. Moreover, $\mathbf{B}_{2 \mathrm{n}}$ was shifted $1 \mathrm{~cm}$ at a time in the $-x$ direction (left) until it reached $\mathbf{B}_{21}$. This process was repeated for all subsequent rows in this 'serpent-like' fashion until the entire IQM sensitive area was irradiated. Response maps were generated for 6, 10 and $15 \mathrm{MV}$ beams and normalized per MU. The mean signal value of five measurements per beamlet was used to be accounted for the variance in the data. All signals were well within $2 \%$ error of the mean as calculated in an Excel spreadsheet. The IQM automatically corrects signals for temperature and pressure variations over time using the chamber's integrated thermometer and barometer.

\section{Elekta iCOM Customer Acceptance Test (iCOMCAT)}

Since the IQM has a spatially sensitive area of $\sim 26 \times 26 \mathrm{~cm}^{2}[6,17]$, the total number of elementary segments $(\mathrm{N})$ required to irradiate the entire chamber for an elementary square segment of side length $L$ shifted by a step size of $s \mathrm{~cm}$ in $x$ and $y$ directions is:

$$
N=\left[1+\frac{(26-L)}{s}\right]^{2}
$$

Equation 2 gives the number of elementary segments, which is shown in Table 1, for the given segment size shifted by a step size of 1 $\mathrm{cm}$. iCOM Customer Acceptance Test (iCOMCAT) software (version 13.0.0.0), which is shown in Figure 2 (left panel), was used to automate the process of irradiating and shifting the regular segments over the chamber sensitive area as presented in Figure 1. iCOMCAT is an Elekta application enabling an external system to transmit a treatment prescription to the treatment control system [18].

Segments prepared in iCOMCAT were exported to the treatment control system via external prescription, which is an interface for the delivery of radiation prescribed by an external record-and-verify system. Elementary segments were delivered in rows in a stepand-shoot manner, with every iCOM file prepared to deliver $(26-\mathrm{L}+1)$ segments per row for the same number of rows in total.

Due to the large number of segments delivered, the number of pre-set MUs per segment was limited to 35 MUs. Figure 2 (right panel) shows the IQM monitoring interface, showing

Table 1: Number of segments and MUs required irradiating the IQM sensitive area for each segment size.

\begin{tabular}{cccc} 
Segment size $\left.\mathbf{( c m}^{2}\right)$ & Step size, $\mathbf{s}(\mathbf{c m})$ & Number of segments $\mathbf{( N )}$ & MUs \\
\hline $1 \times 1$ & 1 & 676 & 23660 \\
\hline $2 \times 2$ & 1 & 625 & 21875 \\
\hline $3 \times 3$ & 1 & 576 & 20160 \\
\hline $5 \times 5$ & 1 & 484 & 16940 \\
\hline $7 \times 7$ & 1 & 400 & 14000 \\
\hline Total & 1 & 2761 & 96635
\end{tabular}


segment-by-segment as well as cumulative IQM signals for a single row of $3 \times 3 \mathrm{~cm}^{2}$ elementary segments. A dose rate of $400 \mathrm{MU} /$ min was used.

\section{Source (signal) obscurity}

Are the above response maps sufficient, and can one conclude that more complex segments are the simple sum of smaller segments? The problem is that a larger segment 'sees' a 'different' scattering source e.g., some scattered radiation that would not have gone through a smaller segment might now do since its 'neighbor' is open (see Figure 3). Larger segment responses would reduce small segment source (signal) obscurity. As a result, larger segments might produce slightly higher signals than the sum of smaller segment signals. This is why larger elementary segments were used to reduce the source obscurity effect.

In Figure 3, since both beams are attenuated in segment 1 and segment 2 , the sum of the two segment signals would yield zero whilst the signal of the combined segments as shown in (c) would yield a non-zero signal value.

\section{Predicting the IQM signal/MU for} an arbitrary segment

From the measured IQM response for a set of small segments shifted over the chamber sensitive area, the second part of this study is

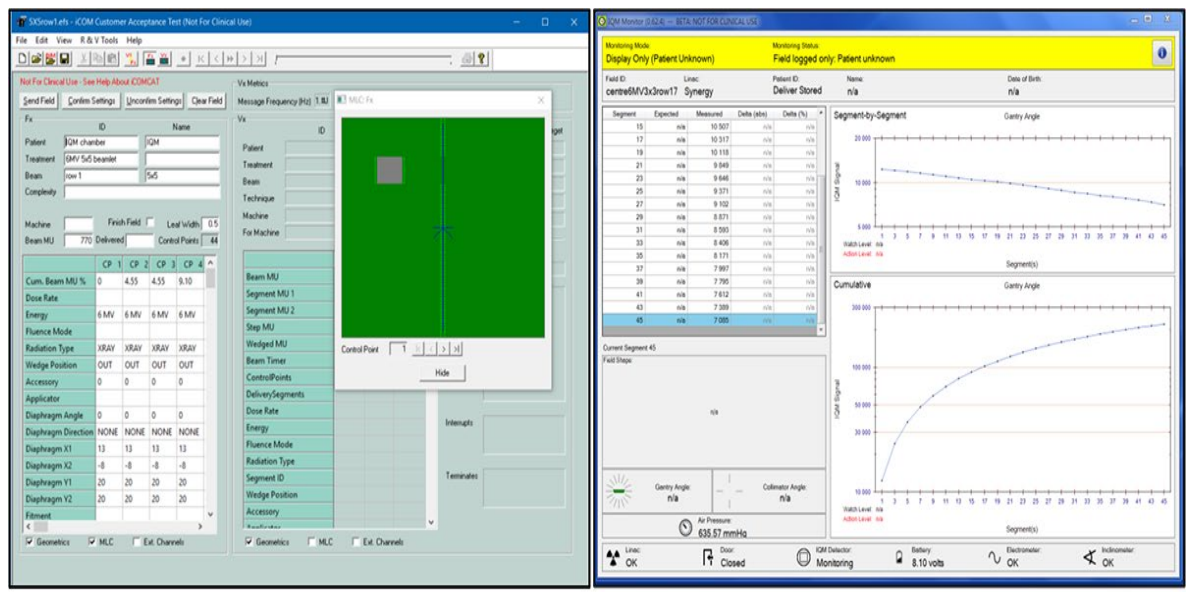

Figure 2: (Left panel) iCOMCAT interface showing the first elementary segment $\mathbf{B}_{11}$ of size $5 \times$ $5 \mathrm{~cm}^{2}$ and the input parameters were used to create the segment. The right panel shows the IQM monitoring interface showing segment-by-segment as well as cumulative IQM signals for a single row of $3 \times 3 \mathrm{~cm}^{2}$ elementary segments.
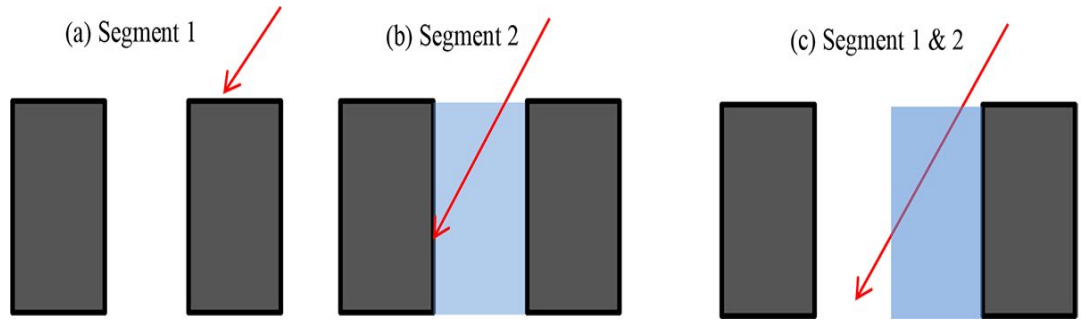

Figure 3: Diagrammatic representation of small segment source obscurity. Scattered radiation in two small adjacent segments 1 and 2 delivered separately is attenuated by MLC blocks as shown in (a) \& (b), but the same radiation could be transmitted through one larger segment in (c). The red arrows represent scattered radiation. 
the prediction of the signal per MU (S) for an arbitrary segment shape and location on the IQM chamber plane for a given beam energy. Arbitrary irregular segments were decomposed into a set of smaller regular segments by first extracting the largest and then the second largest segment etc., until the original segment area was extracted (see example in Figure 4). More irregular clinical segment decomposition may require smaller elementary beamlets. The measured smaller segment signals were obtained from their respective response maps and added together to determine if it would match the measured signal from the original irregular segment. To test the validity of this method, the IQM signal $\left(S^{\prime}\right)$ was measured and evaluated against the predicted signal (S) to determine if other factors needed to be considered for good signal agreement (within $2 \%$ ). This was repeated for a set of regular and irregular segments at 6,10 and $15 \mathrm{MV}$. If the assumption is correct, the predicted signal/ MU would be measurable for any complex segment presented.

\section{In-air spatial distribution of linac fluence}

Since the linac fluence profile can influence IQM signals, it is essential to determine the machine output variation across the field. The spatial distribution of the linac radiation fluence was measured with self-developing Gafchromic EBT3 film due to its high spatial resolution. A $6 \mathrm{MV}$ open photon field of size $15 \times 15 \mathrm{~cm}^{2}$ was projected onto the centre of a $17 \times 17 \mathrm{~cm}^{2}$ film piece placed at the same height as the IQM chamber plane when attached to the linac accessory holder. The distance from the back of the Elekta Synergy Y-diaphragm to the radiation source is $50.9 \mathrm{~cm}$ [19], and the IQM is positioned $4.5 \mathrm{~cm}$ below the diaphragm [20]. This means that the IQM sourceto-surface distance (SSD) is $55.4 \mathrm{~cm}$. The film was supported at this position by a cardboard box and exposed to 500 MUs before being digitally scanned with an HP Colour LaserJet MFP M277n flatbed scanner.

A 24-hour post-irradiation waiting period was observed for proper film development and stabilization due to post-irradiation polymerization [21, 22]. Five blank scans were performed to warm up the lamp and scanner bed. ImageJ was then used to extract the fluence matrix (optical density (OD)) data from the irradiated film. The darkening or OD of the film is relative to the particle lateral distribution across the field in a plane perpendicular to the beam central axis (CAX). Planar fluence distributions can also be derived from BEAMnrc phase space parameters of a beam generated from the radiation machine using BEAMDP software [23].

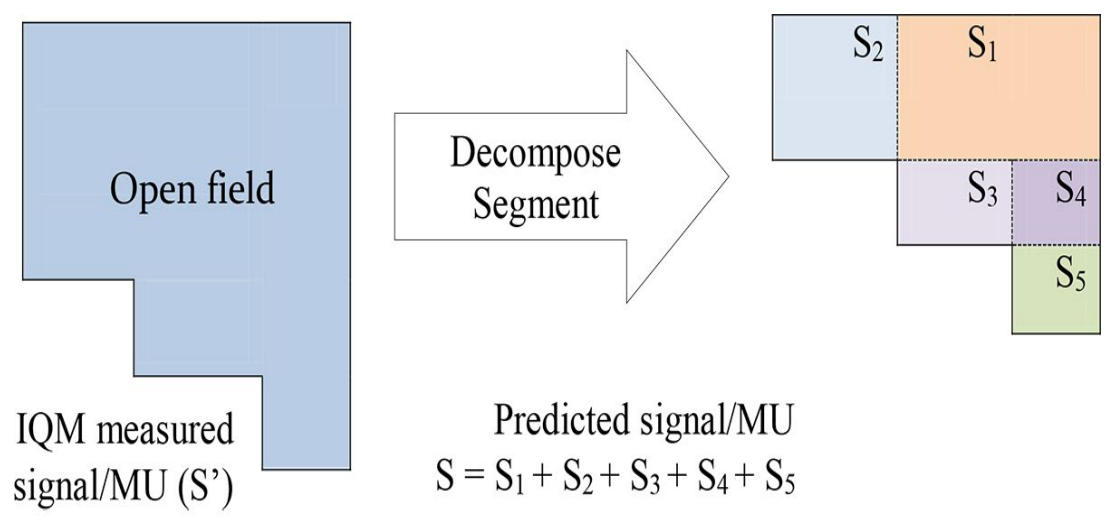

Figure 4: Segmentation of a field into a set of smaller regular segments to predict the signal per $\mathrm{MU}$ for the irregular segment on the left. Signals are measured in arbitrary units (counts). 
Results

\section{IQM response maps}

The spatial response function of the chamber describes the relative chamber response for an elementary segment at a given position with the chamber mounted to the collimator assembly. This section presents the IQM response maps for various segment sizes and photon energies. Mathematica was used to generate the plots. Figure 5 shows a 3D representation of IQM response maps for $1 \times 1 \mathrm{~cm}^{2}$ elementary segments at 6 and $10 \mathrm{MV}$. The relative (rel.) signal is the absolute signal (in counts), normalized per MU. (The response maps for $2 \times$ $2 \mathrm{~cm}^{2}$ and $5 \times 5 \mathrm{~cm}^{2}$ elementary segments are not shown).

IQM inserts are shown at the bottom right corner of Figures 5, 6 and 7 to illustrate the orientation of the chamber relative to the re-
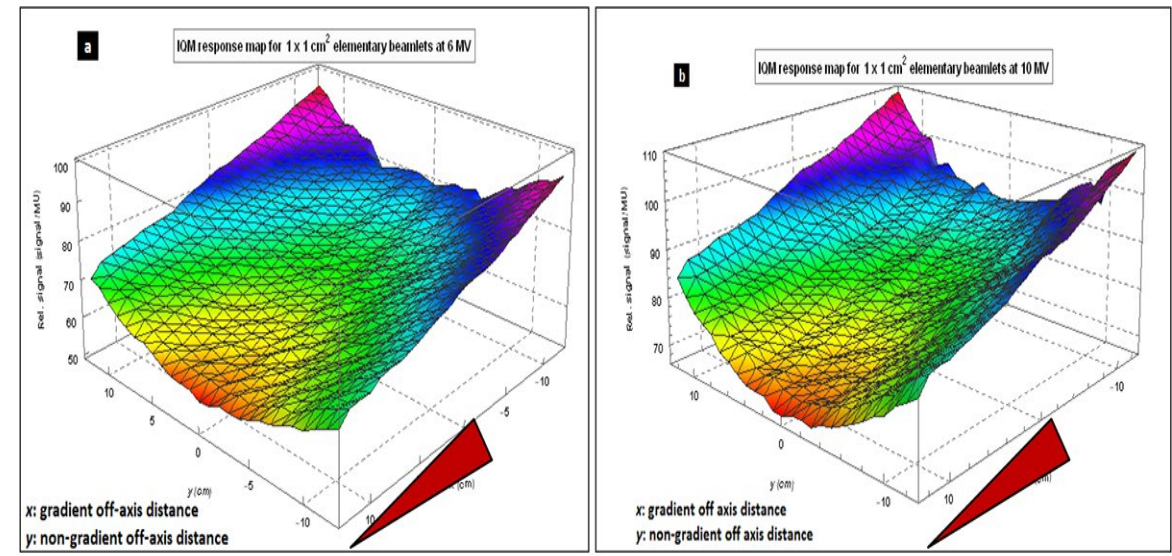

Figure 5: 3D representation of IQM response maps for $1 \times 1 \mathrm{~cm}^{2}$ elementary segments at (a) 6 MV and (b) $10 \mathrm{MV}$. The $\mathrm{x}$-axis is along the IQM gradient direction (as shown by the gradient indicator, meaning where the ionization chamber air volume becomes thicker) whilst $y$-axis is along the non-gradient direction.
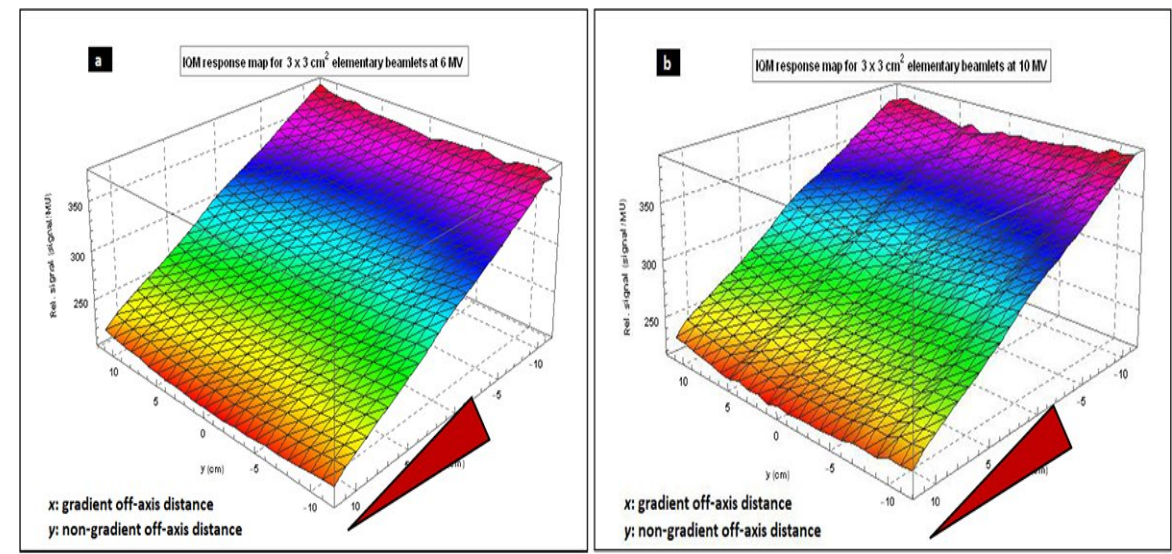

Figure 6: 3D representation of IQM response maps for a $3 \times 3 \mathrm{~cm}^{2}$ elementary segment size at (a) $6 \mathrm{MV}$ and (b) $10 \mathrm{MV}$. The $\mathrm{x}$-axis is along the IQM gradient direction whilst $y$-axis is along the non-gradient direction. 


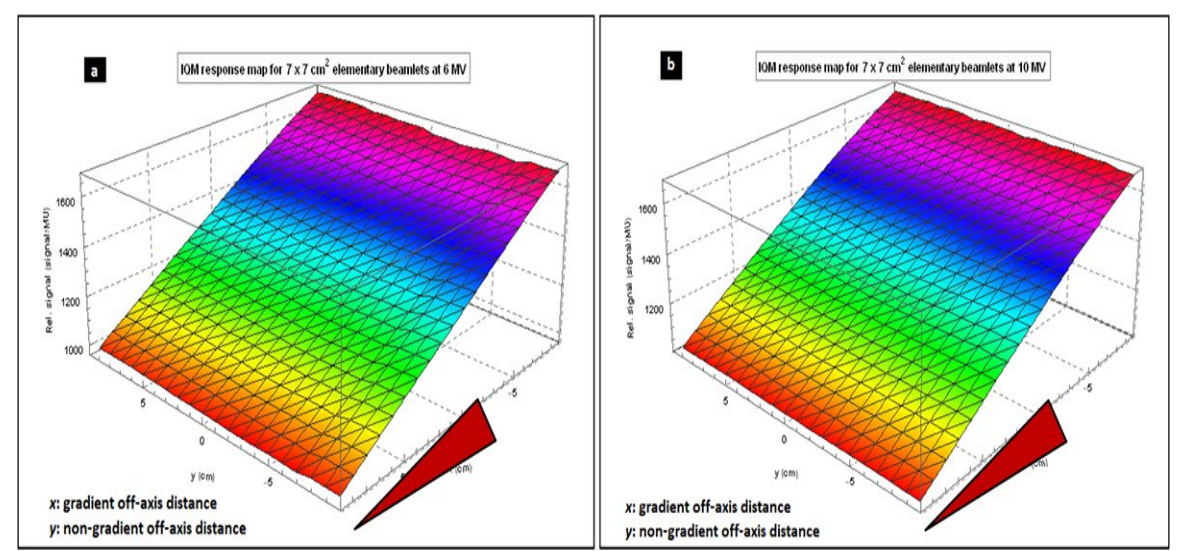

Figure 7: 3D representation of IQM response maps for a $7 \times 7 \mathrm{~cm}^{2}$ elementary segment size at (a) $6 \mathrm{MV}$ and (b) $10 \mathrm{MV}$. The $\mathrm{x}$-axis is along the IQM gradient direction whilst $\mathrm{y}$-axis is along the non-gradient direction.

sponse maps. Figure 6 shows a 3D representation of IQM response maps for $3 \times 3 \mathrm{~cm}^{2} \mathrm{el}-$ ementary segments at 6 and $10 \mathrm{MV}$. Note that the response maps generated by $1 \times 1 \mathrm{~cm}^{2}$ elementary segments appear to be folded whilst response maps become flatter or more uniform as the segment size increases due to a smoothing effect of the larger segment.

Figure 7 shows a $3 \mathrm{D}$ representation of IQM response maps for $7 \times 7 \mathrm{~cm}^{2}$ elementary segments at 6 and $10 \mathrm{MV}$. Note that the response maps appear to be almost flat due to increased smoothing effects compared to the smaller segment response maps above.

\section{Calculated signals for regular segments}

Five signal measurements were performed for each segment size and energy to ensure signal constancy and the average was used. The percentage error of these signals was less than $0.2 \%$ from the mean. The predicted signal $(S)$ was calculated from the response maps using equation 3 below:

$$
S=\sum_{i=1}^{n} S_{i}
$$

Where $n$ is the number of elementary segments, which is decomposed from the field and $\mathrm{S}_{i}$ is the signal for each segment obtained from the relevant IQM signal response map. Table 2 shows measured and calculated signals for regular fields decomposed into several segment sizes at 6,10 and $15 \mathrm{MV}$. The standard deviation was calculated as a measure of noise in the data.

The segments $1 \times 1,2 \times 2$ and $3 \times 3 \mathrm{~cm}^{2}$ used in Table 2 to reconstruct a $5 \times 5 \mathrm{~cm}^{2}$ field produced smaller signals due to reduced scatter in smaller segments. The $5 \times 5 \mathrm{~cm}^{2}$ field was made up with these three smaller regular segments as illustrated in Figure 8(a).

Smaller segments, which were $\leq 2 \times 2 \mathrm{~cm}^{2}$, should at this stage be avoided until a method of correcting for their inaccuracies in the contribution to the larger segments has been laid down in order to get a better match between predicted and measured signals. However, smaller segments are inevitably required to 'fill up' the gaps during field reconstruction as shown in Figure 8(a). Other beam arrangements of segments can also be used to reconstruct the same field, for example, using $1 \times 1$ $\mathrm{cm}^{2}$ segments as shown in Figure 8(b). Other segment arrangements can also be used for the decomposition of the $10 \times 10,15 \times 15$ and $20 \times 20 \mathrm{~cm}^{2}$ fields besides the ones used in Table 2 . When the field is shifted off-axis (away 
Effect of Segment Size and Location on IQM Signals

Table 2: Measured and calculated signals for regular fields at 6, 10 and $15 \mathrm{MV}$ and different segment sizes.

\section{REGULAR FIELDS}

\begin{tabular}{|c|c|c|c|c|c|c|}
\hline $\begin{array}{c}\text { Energy } \\
\text { (MV) }\end{array}$ & $\begin{array}{l}\text { Field size } \\
\qquad\left(\mathrm{cm}^{2}\right)\end{array}$ & $\begin{array}{c}\text { *Meas. } \\
\text { signal, S' } \\
\text { (counts/MU) }\end{array}$ & $\begin{array}{c}\text { Standard } \\
\text { Devia- } \\
\text { tion }(\sigma)\end{array}$ & $\begin{array}{c}{ }^{* *} \text { Calc. } \\
\text { signal, } \mathrm{S} \\
\text { (counts/MU) }\end{array}$ & $\begin{array}{c}\text { Elementary } \\
\text { segment }\left(\mathrm{cm}^{2}\right)\end{array}$ & $\begin{array}{l}\text { Rel. error } \\
\text { (\%) }\end{array}$ \\
\hline \multirow{11}{*}{6} & \multirow{3}{*}{$5 \times 5$} & \multirow{3}{*}{682.63} & \multirow{3}{*}{84.77} & 621.40 & $1 \times 1$ & 8.96 \\
\hline & & & & 646.35 & $1 \times 1,2 \times 2$ & 5.31 \\
\hline & & & & 651.50 & $1 \times 1,2 \times 2,3 \times 3$ & 4.56 \\
\hline & \multirow{3}{*}{$10 \times 10$} & \multirow{3}{*}{2620.34} & \multirow{3}{*}{94.92} & 2671.44 & $5 \times 5$ & 1.95 \\
\hline & & & & 2536.49 & $2 \times 2,3 \times 3$ & 3.20 \\
\hline & & & & 2693.71 & $1 \times 1,3 \times 3,7 \times 7$ & 2.80 \\
\hline & \multirow{3}{*}{$15 \times 15$} & \multirow{3}{*}{5841.89} & \multirow{3}{*}{88.24} & 5951.72 & $5 \times 5$ & 1.88 \\
\hline & & & & 5701.68 & $3 \times 3$ & 2.40 \\
\hline & & & & 5911.99 & $3 \times 3,5 \times 5,7 \times 7$ & 1.20 \\
\hline & \multirow{2}{*}{$20 \times 20$} & \multirow{2}{*}{10321.24} & \multirow{2}{*}{65.57} & 10528.70 & $5 \times 5$ & 2.00 \\
\hline & & & & 10512.18 & $2 \times 2,3 \times 3,7 \times 7$ & 1.85 \\
\hline \multirow{11}{*}{10} & \multirow{3}{*}{$5 \times 5$} & \multirow{3}{*}{707.76} & \multirow{3}{*}{66.02} & 642.65 & $1 \times 1$ & 9.20 \\
\hline & & & & 662.46 & $1 \times 1,2 \times 2$ & 6.40 \\
\hline & & & & 670.11 & $1 \times 1,2 \times 2,3 \times 3$ & 5.32 \\
\hline & \multirow{3}{*}{$10 \times 10$} & \multirow{3}{*}{2711.12} & \multirow{3}{*}{73.84} & 2759.92 & $5 \times 5$ & 1.80 \\
\hline & & & & 2617.59 & $2 \times 2,3 \times 3$ & 3.45 \\
\hline & & & & 2639.28 & $1 \times 1,3 \times 3,7 \times 7$ & 2.65 \\
\hline & \multirow{3}{*}{$15 \times 15$} & \multirow{3}{*}{6025.10} & \multirow{3}{*}{42.21} & 6005.68 & $3 \times 3$ & 2.80 \\
\hline & & & & 6140.78 & $5 \times 5$ & 1.92 \\
\hline & & & & 6103.43 & $3 \times 3,5 \times 5,7 \times 7$ & 1.30 \\
\hline & \multirow{2}{*}{$20 \times 20$} & \multirow{2}{*}{10571.32} & \multirow{2}{*}{60.69} & 10777.46 & $5 \times 5$ & 1.95 \\
\hline & & & & 10759.49 & $2 \times 2,3 \times 3,7 \times 7$ & 1.78 \\
\hline \multirow{11}{*}{15} & \multirow{3}{*}{$5 \times 5$} & \multirow{3}{*}{710.77} & & 639.69 & $1 \times 1$ & 10.0 \\
\hline & & & 54.61 & 669.19 & $1 \times 1,2 \times 2$ & 5.85 \\
\hline & & & & 673.53 & $1 \times 1,2 \times 2,3 \times 3$ & 5.24 \\
\hline & & & & 2791.11 & $5 \times 5$ & 2.0 \\
\hline & $10 \times 10$ & 2736.38 & 53.70 & 2648.82 & $2 \times 2,3 \times 3$ & 3.20 \\
\hline & & & & 2811.36 & $1 \times 1,3 \times 3,7 \times 7$ & 2.74 \\
\hline & & & & 5951.29 & $3 \times 3$ & 2.50 \\
\hline & $15 \times 15$ & 6103.89 & 61.45 & 6219.86 & $5 \times 5$ & 1.90 \\
\hline & & & & 6164.32 & $3 \times 3,5 \times 5,7 \times 7$ & 0.99 \\
\hline & & & & 10924.65 & $5 \times 5$ & 2.00 \\
\hline & & & 5 & 10905.37 & $2 \times 2,3 \times 3,7 \times 7$ & 1.82 \\
\hline
\end{tabular}

$*$ Meas. $=$ Measured, ${ }^{*}$ Calc. $=$ Calculated 

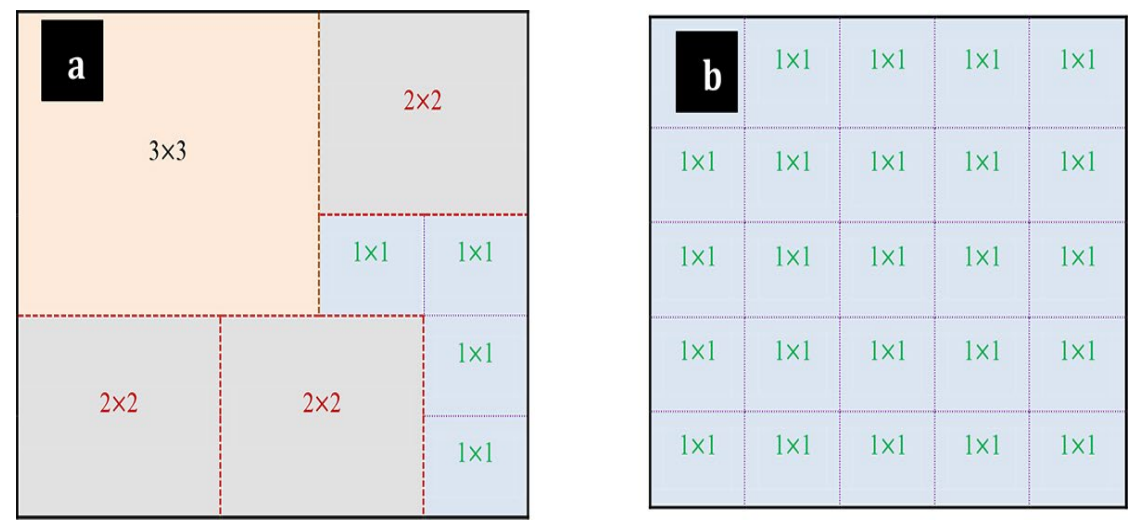

Figure 8: Panel (a) illustrates the decomposition of a $5 \times 5 \mathrm{~cm}^{2}$ field into $1 \times 1,2 \times 2$ and $3 \times 3 \mathrm{~cm}^{2}$ segments. Panel (b) shows an alternative $1 \times 1 \mathrm{~cm}^{2}$ segment arrangement, which, however, was less accurate in signal prediction.

from the IQM centre), there is a substantial signal change, depending on its exact location. The highest signals are measured on the thickest part of the chamber with the largest air volume and smaller signals on the thinner part with lower signal response as observed in the sensitivity maps.

Dependence of calculated signal on segment size

Figure 9 shows gradient and non-gradient CAX profiles for various segment sizes at $15 \mathrm{MV}$. The orientation of the chamber rela- tive to the direction of signal measurement is shown. Error bars are drawn on one profile for visibility. The profile shapes are consistent with the response maps. Gradient profiles have a concave shape; the highest signals appear close to the gradient peripheral regions of the chamber and the lowest signals on the gradient central axial direction. This is not an IQM artefact and due to the original shape of the exit fluence profile generated by the linac. For gradient profiles, larger segments produce steeper profiles than smaller segments. This trend is
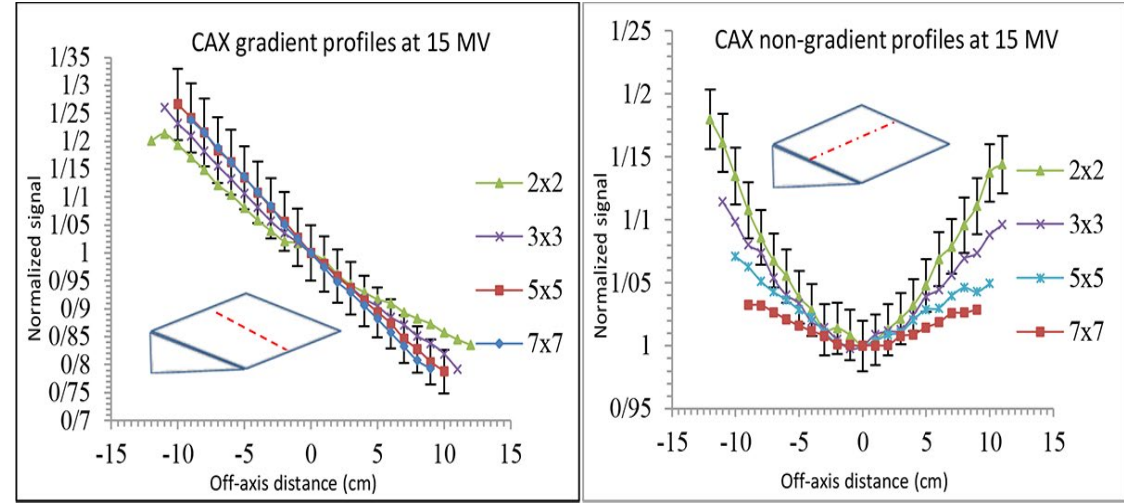

Figure 9: Variation of calculated signals on the segment size for gradient and non-gradient profiles at $15 \mathrm{MV}$. The orientation of the IQM is shown. Profiles were drawn along the red dotted line on the chamber. 
the same for other energies in this study.

Energy dependence of measured and calculated signals

To illustrate the dependence of electronic signals on the beam energy, Figure 10 shows comparisons of profiles for $7 \times 7 \mathrm{~cm}^{2}$ segments at different beam energies.

For non-gradient profiles (right panel) highenergy beams yield larger signals whilst for gradient profiles, there is little dependence of beam profiles on energy. From Table 2, increasing the beam energy from 6 to $10 \mathrm{MV}$ and from 10 to $15 \mathrm{MV}$ for a $10 \times 10 \mathrm{~cm}^{2}$ field only increases the calculated signal by 3.3 and $1.1 \%$, respectively. These percentages are roughly in the same range for other regular fields in Table
2. This trend is also the same for other segments sizes (not shown). Some random points on the IQM for non-gradient profiles exhibit minor over- and under-response and give fluctuating output signals. This is caused by minor fluctuations in linac beam fluence causing the signal fluctuations as in Figure 10 (a).

\section{Calculated signals for arbitrary irregular segments}

Figure 11 shows a representation of three irregular segments used and how they were decomposed into smaller elementary segments to calculate the predicted signal.

Fields $\mathbf{A}$ and $\mathbf{B}$ were of different shapes but almost of the same size and placement (rough-

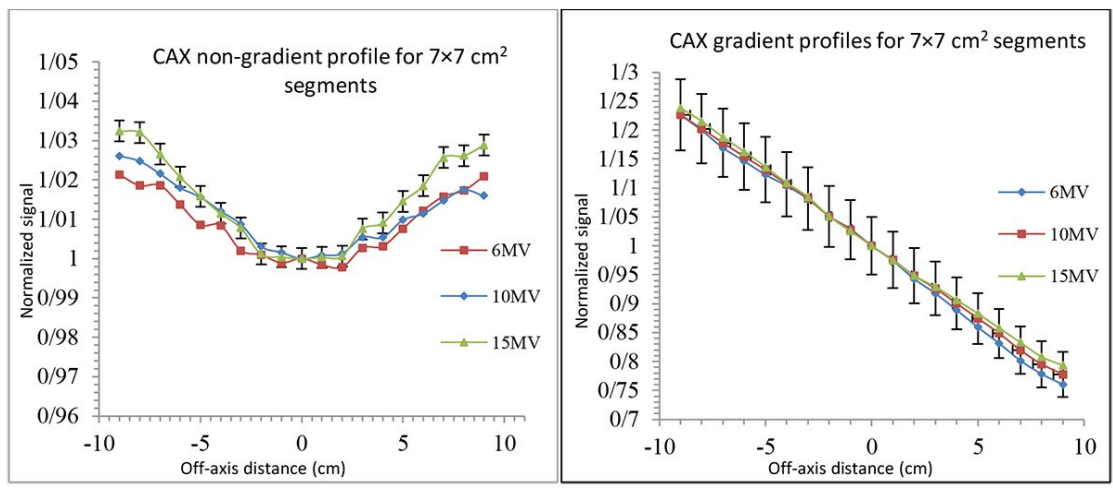

Figure 10: Dependence of measured signals on the beam energy for gradient and non-gradient profiles. The profiles are normalized to the centre of the chamber. Error bars are only drawn on one profile for visibility.
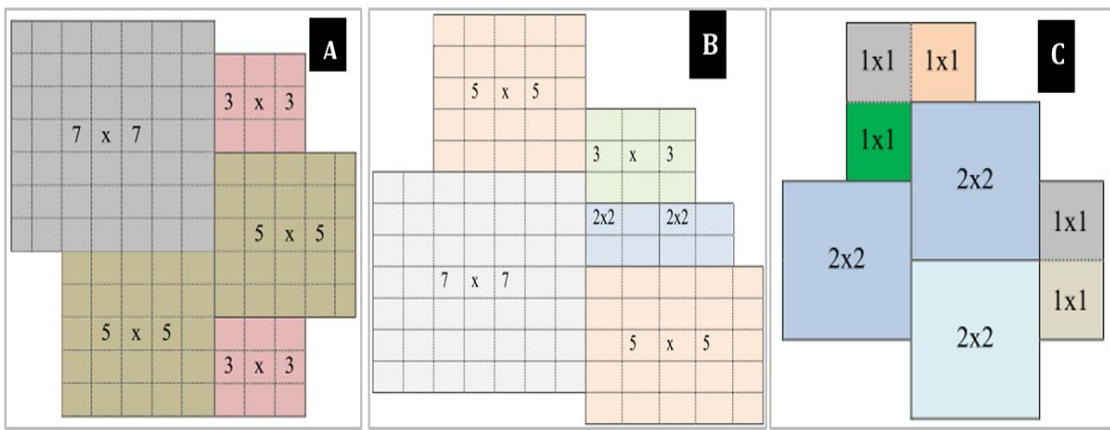

Figure 11: Representation of 3 irregular fields and how they were decomposed into smaller segments to calculate the predicted signal. The segments are drawn to scale. The fields were arbitrarily created 
ly at the centre) of the IQM - whilst field $\mathbf{C}$ is a relatively small segment projected close to the gradient edge of the device. The fields were made with different shapes, sizes and location in order to investigate the influence of these factors on the predicted signals. Table 3 shows measured and calculated signals for 3 different irregular fields (A, B and C) in Figure 11 at 6, 10 and $15 \mathrm{MV}$. The standard deviation was also calculated to quantify the noise in the data.

It is evident from Table 3 that small segments signals should be corrected to achieve a better match between predicted and measured signals. A method of correcting for their inaccuracies in the contribution to the larger segments will however be laid down in a future study.

\section{$2 D$ exit fluence map of the linear accelerator}

The spatial shape of the linac fluence distri- bution in air as measured with EBT3 film is shown in Figure 12. This fluence was extracted from the irradiated part of the film and plotted by ImageJ software. The plot was zoomed to illustrate the high fluence in the matrix field edges. This explains the over-and-under response in the response maps and signal profiles as discussed earlier.

The increase of fluence with distance from the central axis in Figure 12 is induced by the shape of the flattening filter inside the treatment head, which improves the dose at the isocentre plane.

\section{Discussion}

The influence of beam segment size and displacement as projected onto the IQM on its output signal was determined. This is a precursor for a second part of this study to determine if the output signal per MU for a complex segment shape could be calculated from a set of smaller regular segments of which the output

Table 3: Measured and calculated signals calculated using different combinations of elementary segments to reconstruct the open fields for 3 different irregular fields at 6, 10 and $15 \mathrm{MV}$.

\begin{tabular}{|c|c|c|c|c|c|c|}
\hline $\begin{array}{c}\text { Energy } \\
\text { (MV) }\end{array}$ & $\begin{array}{l}\text { Field size } \\
\quad\left(\mathrm{cm}^{2}\right)\end{array}$ & $\begin{array}{c}\text { *Meas. } \\
\text { signal, S' } \\
\text { (counts/MU) }\end{array}$ & $\begin{array}{c}\text { Standard } \\
\text { Deviation } \\
(\sigma) \\
\end{array}$ & $\begin{array}{c}{ }^{* *} \text { Calc. } \\
\text { signal, } \mathrm{S} \\
\text { (counts/MU) }\end{array}$ & $\begin{array}{c}\begin{array}{c}\text { Elemen- } \\
\text { tary segment } \\
\left(\mathrm{cm}^{2}\right)\end{array} \\
\end{array}$ & $\begin{array}{c}\text { Rel. } \\
\text { error (\%) }\end{array}$ \\
\hline \multirow{3}{*}{6} & $\mathbf{A}$ & 3028,50 & 42.55 & 3093.61 & $3 \times 3,5 \times 5,7 \times 7$ & 2.15 \\
\hline & B & 2870,18 & 42.99 & 2969.49 & $\begin{array}{c}2 \times 2,3 \times 3 \\
5 \times 5,7 \times 7\end{array}$ & 3.46 \\
\hline & C & 446,65 & 14.02 & 409.31 & $1 \times 1,2 \times 2$ & 8.36 \\
\hline \multirow{3}{*}{10} & $\mathbf{A}$ & 3083,07 & 56.94 & 3152.44 & $3 \times 3,5 \times 5,7 \times 7$ & 2.25 \\
\hline & B & 2926,84 & 62.86 & 3024.60 & $\begin{array}{c}2 \times 2,3 \times 3 \\
5 \times 5,7 \times 7\end{array}$ & 3.34 \\
\hline & C & 454,46 & 15.72 & 409.70 & $1 \times 1,2 \times 2$ & 9.85 \\
\hline \multirow{3}{*}{15} & $\mathbf{A}$ & 3090,75 & 74.62 & 3176.67 & $3 \times 3,5 \times 5,7 \times 7$ & 2.78 \\
\hline & B & 2918,82 & 66.52 & 3030.32 & $\begin{array}{c}2 \times 2,3 \times 3 \\
5 \times 5,7 \times 7\end{array}$ & 3.82 \\
\hline & C & 461.30 & 17.96 & 419.78 & $1 \times 1,2 \times 2$ & 9.00 \\
\hline
\end{tabular}

A, $\mathbf{B}$ and $\mathbf{C}$ are the field segments in Figure 12.

*Meas. $=$ Measured, $* *$ Calc. $=$ Calculated 


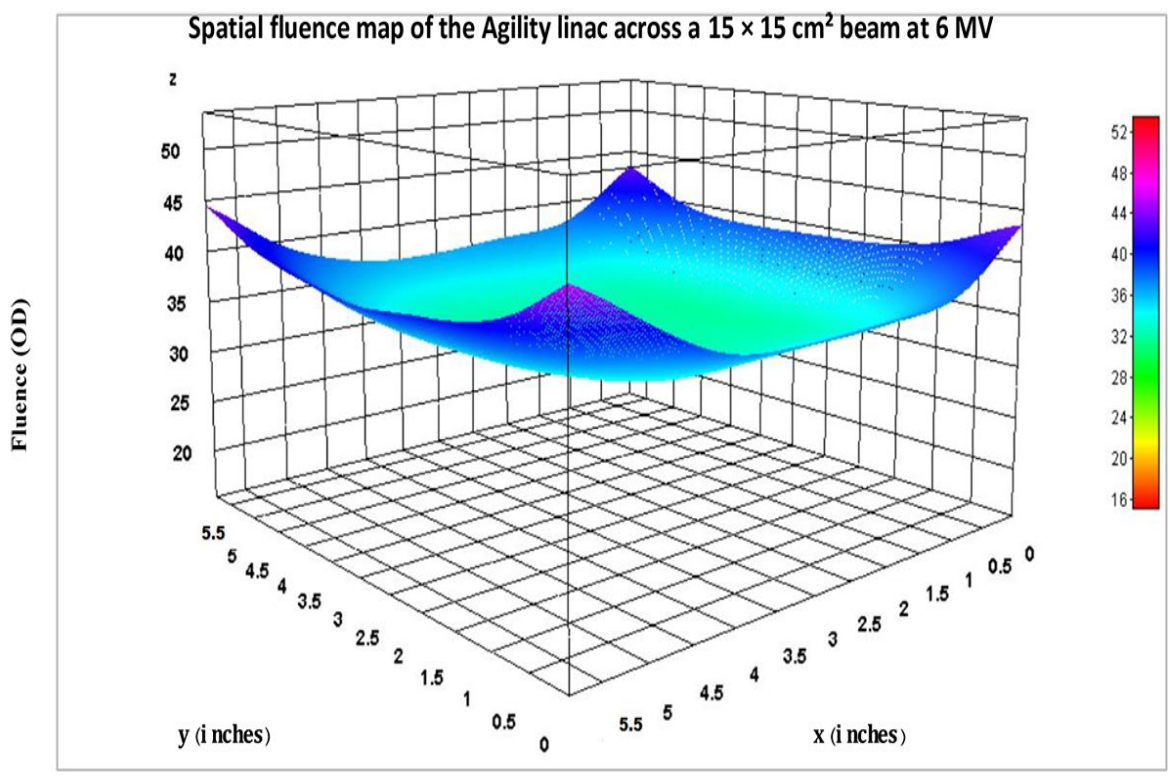

Figure 12: 3D surface plot of the optical density fluence profile of the Agility linac for a $15 \times 15$ $\mathrm{cm}^{2}$ field at $6 \mathrm{MV}$. From the colour gradient, the map is approximately uniform but with higher fluence on the peripheral regions. The OD is scaled to visualize the effects of the flattening filter on the fluence map more clearly.

per MU is known. Thus, factors such as beam energy, segment combination and segment shape were considered. IQM response maps were generated for regular segment shapes over the total sensitive area of the IQM at 6, 10 and $15 \mathrm{MV}$. CAX profiles taken in the wedge gradient direction of the IQM and across were normalized to the centre of the chamber and also investigated. Measured and calculated signals for regular and irregular segments were also determined using different combinations of smaller regular segments. The fluence spatial distribution of the Synergy linac was also measured to determine the output variation across the field.

It was found that the calculated signal per MU was lower than the measured signal when smaller segments $\left(\leq 3 \times 3 \mathrm{~cm}^{2}\right)$ were used. This can be attributed to the source obscurity effect, which were more pronounced for smaller segments as well as scattered photons that were shielded off by the MLC and jaws in smaller elementary segments. Response maps generated by smaller elementary segments have a folded profile whilst response maps become flatter or more uniform as the segment size increases due to a smoothing effect of the larger segment. Higher signals were measured on the thicker part of the ionization chamber. A wider separation distance between the plates increases the mass of gas contained. More mass will result in more ions, which were created as radiation passes through the volume, increasing the signal measured. Thus, greater separation will result in a greater signal and vice versa $[15,16]$.

Non-gradient profiles have a concave shape, with the highest signals appearing close to the peripheral regions of the chamber and the lowest signals along the gradient central axial direction. This is not an IQM artefact and also could have resulted from the original shape of the fluence profile produced by the linac. The response maps are however virtually symmetrical, especially for larger elementary segments, which is consistent with the geometrical symmetry in the wedge shape of the chamber and the symmetry in the fluence pro- 
file of the linear accelerator. The increase of fluence with distance from the central axis is induced by the shape of the flattening filter inside the treatment head, improving the dose at the isocentre plane.

To ensure accuracy, 10 signal measurements were taken for each segment and the average signal was used. The noise in the data was also quantified using the standard deviation. Using different elementary segment sizes and combinations results in different calculated signals due to source obscurity and radiation scatter from field edges of elementary segments used to reconstruct the larger segment under consideration. Based on the findings, for larger segments, larger elementary segments must be used to calculate the output signal per MU. Smaller segments, which were $\leq 2 \times 2 \mathrm{~cm}^{2}$, should at this stage be avoided until a method of correcting for their inaccuracies in the contribution to the larger segments laid down in order to get a better match between predicted and measured signals. This will however be addressed in a future study.

High energy beams have more energetic secondary electrons that can impart their energy in air, causing ionizations that can increase the IQM signal, and hence the observed dependency of the signals on energy. The signal increases due to a slight increase in the beam energy.

Smaller segments produce less scattered radiation. From Figure 11, segment $\mathbf{A}$ has a total area of $117 \mathrm{~cm}^{2}$, segment $\mathbf{B}$ is $116 \mathrm{~cm}^{2}$ and segment $\mathbf{C}$ is $17 \mathrm{~cm}^{2}$. Due to their similar sizes, segments $\mathbf{A}$ and $\mathbf{B}$ gave almost the same signals, whilst the smallest segment $\mathbf{C}$ gave the smallest signal. This however, would depend on the position and displacement of these segments relative to the central axis. The smallest segment gave the greatest signal calculation error as seen in Table 3. Segment $\mathbf{C}$ is composed of $1 \times 1$ and $2 \times 2 \mathrm{~cm}^{2}$ beamlets whilst segments $\mathbf{B}$ and $\mathbf{C}$ are composed of $2 \times 2,3 \times 3,5 \times 5$ and $7 \times 7 \mathrm{~cm}^{2}$ beamlets, therefore more scatter is expected in the lat- ter. Increasing the beam energy from 6 to 10 $\mathrm{MV}$ and from 10 to $15 \mathrm{MV}$ for field A only increased the calculated signal by 1.90 and $0.77 \%(<2 \%)$, respectively. These percentages are roughly in the same range for other irregular fields in Table 3 . The relative errors involved in calculating segment signals increase significantly, as the field size gets smaller and more irregular, and hence reconstructed using even smaller segments where signal obscurity and reduced scatter is significant.

\section{Conclusion}

IQM signals for irregular segments can, in principle, be calculated beforehand from a set of smaller regular segments extracted from the segment under consideration. The requirement is that for each smaller regular segment, its signal must be known at the location on the IQM form measured sensitivity maps as found in Figures 5, 6 and 7. Summation of these segment signals can produce the larger segment signal, although signal calculation accuracy becomes poor for smaller irregular fields due to source obscurity and decreased scatter contribution. There is little dependence of gradient profiles on energy and a steeper signal variation for larger segments than smaller segments. Proper choice of elementary segment combination is critical to obtain maximum accuracy in calculating predicted signals. It was established that segment size and location as well as segment combination have the greatest effect on calculated signals whilst a change in beam energy has considerably less impact. Shifting the same field towards the thicker (or thinner) part of the chamber (i.e., in the gradient direction) substantially magnifies (or reduces) the signal due to differences in air volumes, which contained and the subsequent number of ions created as radiation passes and interacts with the medium. It is recommended that the signal calculation for a complex segment be restricted to cases where the segment can be decomposed with a minimum number of smaller segments with sizes $\leq 2 \times 2 \mathrm{~cm}^{2}$ and 
should be avoided at this stage.

\section{Acknowledgment}

This research and the publication thereof is the result of funding provided by the South African Medical Research Council (MRC) in terms of MRC's Flagship Awards Project under the High Energy Advanced Radiation Dosimetry (HARD) sponsorship program SAMRC-RFA-UFSP-01-2013/HARD with funds from the National Treasury under its Economic Competitiveness and Support Package.

\section{Conflict of Interest}

None

\section{References}

1. Islam MK, Norrlinger BD, Smale JR, Heaton RK, Galbraith D, Fan C, et al. An integral quality monitoring system for real-time verification of intensity modulated radiation therapy. Med Phys. 2009;36:5420-8. doi: 10.1118/1.3250859. PubMed PMID: 20095254.

2. Noel CE, Santanam L, Parikh PJ, Mutic S. Process-based quality management for clinical implementation of adaptive radiotherapy. Med Phys. 2014;41:081717. doi: 10.1118/1.4890589. PubMed PMID: 25086527. PubMed PMCID: PMC4119199.

3. Poppe B, Looe HK, Chofor N, Ruhmann A, Harder $D$, Willborn KC. Clinical performance of a transmission detector array for the permanent supervision of IMRT deliveries. Radiother Oncol. 2010;95:15865. doi: 10.1016/j.radonc.2009.12.041. PubMed PMID: 20138379.

4. Stelljes TS, Harmeyer A, Reuter J, Looe HK, Chofor N, Harder D, et al. Dosimetric characteristics of the novel 2D ionization chamber array OCTAVIUS Detector 1500. Med Phys. 2015;42:1528-37. doi: 10.1118/1.4914151. PubMed PMID: 25832043.

5. Chang J, Heaton RK, Mahon R, Norrlinger BD, Jaffray DA, Cho YB, et al. A method for online verification of adapted fields using an independent dose monitor. Med Phys. 2013;40:072104. doi: 10.1118/1.4811204. PubMed PMID: 23822448.

6. Hoffman D, Chung E, Hess C, Stern R, Benedict $S$. Characterization and evaluation of an integrated quality monitoring system for online quality assurance of external beam radiation therapy. $J$ Appl Clin Med Phys. 2017;18:40-8. doi: 10.1002/ acm2.12014. PubMed PMID: 28291937. PubMed PMCID: PMC5689870.

7. Nakaguchi Y, Araki F, Maruyama M, Saiga S. Dose verification of IMRT by use of a COMPASS transmission detector. Radiol Phys Technol. 2012;5:63-70. doi: 10.1007/s12194-011-0137-y. PubMed PMID: 22038312.

8. Thoelking J, Fleckenstein J, Sekar Y, Boggula R, Lohr F, Wenz F, et al. Patient-specific online dose verification based on transmission detector measurements. Radiother Oncol. 2016;119:351-6. doi: 10.1016/j.radonc.2016.04.003.

9. Velthuis JJ, Hugtenburg RP, Cussans D, Perry $M$, Hall $C$, Stevens $P$, et al. The VANILLA sensor as a beam monitoring device for $\mathrm{X}$-ray radiation therapy. Appl Radiat Isot. 2014;83:8-11. doi: 10.1016/j.apradiso.2013.10.007. PubMed PMID: 24215812.

10. Wong JH, Fuduli I, Carolan M, Petasecca M, Lerch ML, Perevertaylo VL, et al. Characterization of a novel two dimensional diode array the "magic plate" as a radiation detector for radiation therapy treatment. Med Phys. 2012;39:2544-58. doi: 10.1118/1.3700234. PubMed PMID: 22559625.

11. Goulet M, Gingras L, Beaulieu L. Real-time verification of multileaf collimator-driven radiotherapy using a novel optical attenuation-based fluence monitor. Med Phys. 2011;38:1459-67. doi: 10.1118/1.3549766.

12. IRT Systems. Integral Quality Monitor Improving Patient Safety. iRT Systems White Paper Presentation; 2014.

13. Islam MK, Norrlinger BD, Galbraith DM, Jaffray DA, Heaton RK, Smale J. Apparatus and methods for real-time verification of radiation therapy. Patent Appl, United States; 2007.

14. Norrlinger B, Islam M. Characterization of A Large-Area Transmission Chamber For Independent Verification Of IMRT Dose Delivery Constancy. Med Phys. 2003;29:1273.

15. DeWerd LA, Wagner LK. Characteristics of radiation detectors for diagnostic radiology. Appl Radiat Isot. 1999;50:125-36. doi: 10.1016/s09698043(98)00044-x. PubMed PMID: 10028632.

16. Seco J, Clasie B, Partridge M. Review on the characteristics of radiation detectors for dosimetry and imaging. Phys Med Biol. 2014;59:R303-47. doi: 10.1088/0031-9155/59/20/R303. PubMed PMID: 25229250.

17. Oderinde OM, Du Plessis F. A new wedgeshaped ionization chamber component module for BEAMnrc to model the integral quality moni- 
Mahuvava C. and Du Plessis F. C. P.

toring system $®$. Radiation Physics and Chemistry. 2017;141:346-51. doi: 10.1016/j.radphyschem.2017.08.005.

18. Kaurin DG, Sweeney LE, Marshall El, Mahendra S. VMAT testing for an Elekta accelerator. J App/ Clin Med Phys. 2012;13:3725. doi: 10.1120/jacmp. v13i2.3725. PubMed PMID: 22402389. PubMed PMCID: PMC5716421.

19. Elekta Limited. Agility ${ }^{\top M}$ and Integrity ${ }^{\top M}$ R3.x. Information for Treatment Planning Systems. Document ID: 1504231 01; 2013.

20. Oderinde OM, Du Plessis FCP. Sensitivity Analysis of the Integral Quality Monitoring System(R) Using Monte Carlo Simulation. Comput Math Methods Med. 2017;2017:7025281. doi: 10.1155/2017/7025281. PubMed PMID: 28928795. PubMed PMCID: PMC5591992.

21. Reinstein LE, Gluckman GR, Meek AG. A rapid colour stabilization technique for radiochromic film dosimetry. Phys Med Biol. 1998;43:2703-8. doi: 10.1088/0031-9155/43/10/001. PubMed PMID: 9814510.

22. Girard F, Bouchard H, Lacroix F. Reference dosimetry using radiochromic film. J Appl Clin Med Phys. 2012;13:3994. doi: 10.1120/jacmp. v13i6.3994. PubMed PMID: 23149793. PubMed PMCID: PMC5718535.

23. Ma CM, Rogers DWO. BEAMDP users manual. NRC Report PIRS-0509 (D), Ottawa: National Research Council of Canada; 1995. 